Proceedings of SALT 22: 383-402, 2012

\title{
No need for a dedicated theory of the distribution of readings of English bare plurals*
}

\author{
Giorgio Magri \\ LLF (CNRS, Université Paris 7)
}

\begin{abstract}
This paper argues that English bare plurals (BPs) uniformly have a plain existential semantics. Their generic/universal reading arises through a mechanism of exhaustification modeled on Spector (2007). The sentence Some firemen are tall triggers the implicature that only some firemen are tall. The variant Firemen are tall triggers the implicature that this "only-some" implicature is false. The universal reading of the BP thus arises through a "not-only-some" implicature. The distribution of existential and generic readings of BPs is thus completely determined by the availability of the "only-some" implicature of the corresponding indefinite, without any need for a dedicated theory of the distribution of readings of BPs.
\end{abstract}

Keywords: bare plurals, scalar implicatures, plural morphology.

\section{Introduction}

English bare plurals (BPs) can have (at least) three readings: generic, existential and Condoravdi's (1997) reading. ${ }^{1}$ The distribution of these readings is constrained by a variety of grammatical factors, such as predicate type (individual- versus stage-level predicates), syntactic scope (within versus outside of VP), information structure (focus versus topic), argument type (subject versus object), and so on. Various authors have thus developed rich, dedicated theories of the distribution of readings of English BPs, that make use of a number of covert operators together with grammatical constraints on their scope, such as the generic operator, Chierchia's (1995) Derived Kind Predication, Heim's (1988) Default Existential Closure, and so on. This paper explores the hypothesis that there is actually no need for a dedicated theory of English BPs: the distribution of the readings of a BP such as firemen is

* I would like to thank Gennaro Chierchia, Danny Fox, Raj Singh and Benjamin Spector for very useful discussion on the material presented in this paper. This material was also presented at CGG 22 (Barcelona, February 2012) and at the LSALAA workshop (Université Paris 8, March 2012); I would like to thank the audiences at those venues for valuable feed-back.

1 In this paper, I ignore the kind reading of BPs, which I take to be derived in a completely different way than the three readings considered here (say, by having the BP denote a kind). 
entirely determined by the availability of the "only-some" implicature triggered by the corresponding overt indefinite some firemen.

My starting point is the observation in Section 2 that the generic reading of BPs behaves as the plurality inference triggered by English plural morphology relative to embedding in environments of different monotonicity. This analogy calls for a unified account. In Section 3, I review Spector's (2007) pragma-semantics for the behavior of the plurality inference and I trivially extend it to BPs. According to this proposal, the plain meaning of a BP such as firemen is always just existential, whereby it is equivalent to the corresponding indefinite some firemen. The BP's universal/generic reading arises through a mechanism of (double) strengthening, that works roughly as follows. The corresponding indefinite some firemen triggers the "only-some" (1st order) scalar implicature. The choice of the BP over the indefinite triggers the "not-only-some" (2nd order) scalar implicature that this "only-some" implicature is false. And this "not-only-some" scalar implicature yields the universal/generic reading. This proposal predicts the availability of a BP's universal/generic reading to correlate with the availability of the "only-some" implicature of the corresponding indefinite. In Section 4, I argue that this prediction is correct, as it amounts to a corollary of Diesing's (1992) framework, namely the fact that a BP can be interpreted universally/generically only when it sits in a position where the corresponding indefinite can be interpreted presuppositionally.

\section{A parallelism between the plurality inference and the generic inference}

In Subsection 2.1, I review from the literature the intriguing behavior of the plurality inference triggered by English plural morphology relative to embedding in environments of different monotonicity. In Subsection 2.2, I then point out that the generic reading of English BPs displays an analogous behavior. This observation calls for a unified account, developed in Section 3.

\subsection{The behavior of the plurality inference under embedding}

Sentence (1a) with the plural indefinite some books triggers the plurality inference (P-inference) that John bought more than one book.

(1) a. John bought some linguistic books.

b. John bought (at least) two linguistic books.

c. John bought a linguistic book.

Thus, sentence (1a) feels equivalent to sentence (1b) with the quantified indefinite (at least) two books and both feel stronger than sentence (1c) with the singular indefinite 
No need for a dedicated theory of English bare plurals

a book. In fact, the latter sentence (1c) would feel true in a scenario where John bought a single linguistic book, contrary to sentences (1a) and (1b).

It is well known that this P-inference behaves surprisingly in downward entailing (DE) environments, such as the scope of negation or the restrictor and the nuclear scope of the determiner no. Sentence (2a) with the plural indefinite ${ }^{2}$ embedded under negation feels equivalent to sentence (2c) with the singular indefinite and both feel stronger than sentence (2b) with a quantified indefinite. In fact, the latter sentence (2b) would feel true in a scenario where John bought a single linguistic book, contrary to the other two sentences (2a) and (2c).

(2) a. John didn't buy any linguistic books.

b. John didn't buy (at least) two linguistic books.

c. John didn't buy a linguistic book.

Indeed, only sentence ( $2 b$ ) with the quantified indefinite can be felicitously continued with but he did buy one as in (3b), while that continuation feels odd in the case of sentences (2a) and (2c) with a plural and a singular indefinite, as in (3a) and (3c).

a. \#John didn't buy linguistic books, but he did buy one.

b. John didn't buy (at least) two linguistic books, but he did buy one.

c. \#John didn't buy a linguistic book, but he did buy one.

In conclusion, the P-inference disappears under negation, raising a compositionality puzzle: sentence (2a) does not mean the negation of sentence (1a), although syntactically the former is indeed just the negation of the latter.

Spector (2007) adds the elegant observation that both the upward entailing (UE) and DE behaviors are visible in the case of embedding in a non-monotonic environment such as the scope of exactly one student, as illustrated in (4).

(4) a. Exactly one student solved some difficult problems.

b. Exactly one student solved (at least) two difficult problems.

c. Exactly one student solved a difficult problem.

Sentence (4a) with the plural indefinite differs from sentence (4c) with the singular indefinite in the same respect that the unembedded sentence (1a) differs from (1c): only (4c) but not (4a) would feel true in a scenario where the unique student who solved difficult problems solved only one of them. In other words, the P-inference is visible in the UE component of the non-monotonic meaning. Furthermore, sentence (4a) with the plural indefinite differs from sentence (4b) with a quantified indefinite

2 From (1a) to (2a), I have switched from some to any because of the PPIness of the former, but I consider the two items as equivalent for all intended purposes. 
in the same respect that the sentence (2a) differs from (2b): only (4b) but not (4a) would feel true in a scenario where, besides the unique student who solved two or more problems, there are a few more students who solved only one. In other words, the $\mathrm{P}$-inference is not visible in the DE component of the non-monotonic meaning.

\subsection{The behavior of the generic inference under embedding}

Sentence (5a) with the BP subject dogs triggers the inference that it is a general property of dogs that they are intelligent. Let me call this inference the generic inference (G-inference). Throughout this paper, I will ignore the issue of the quality of the generic meaning, namely of the proper quantificational force of generics and of their tolerance to exceptions. And I will thus treat generics as equivalent to universals. The issue of the quality of the generic meaning indeed seems orthogonal to the issue of its distribution, which is the focus of this paper. ${ }^{3}$

a. Dogs are intelligent.

b. All dogs are intelligent.

c. Some dogs are intelligent.

With these qualifications, sentence (5a) is equivalent to the universally quantified sentence $(5 \mathrm{~b})$. Indeed, both sentences feel stronger than the existentially quantified sentence $(5 c)$. In fact, the latter sentence $(5 c)$ would feel true in a scenario where less than fifty percent of dogs are intelligent, contrary to (5a) and (5b).

It is well known that the G-inference behaves surprisingly in DE environments (Fodor 1970; von Fintel 1997; Gajewski 2005). Sentence (6a) with the BP embedded under negation feels equivalent to the existentially quantified sentence (6c) and both feel stronger than the universally quantified sentence (6b). In fact, the latter sentence (6b) would feel true in a scenario where half of the dogs are intelligent, contrary to the other two sentences (6a) and (6c).

a. It's false that dogs are intelligent.

b. It's false that all dogs are intelligent.

c. It's false that some dogs are intelligent.

Indeed, only the universally quantified sentence (6b) can be felicitously continued with but many of them are as in (7b), while that continuation feels odd in the case of sentence (6a) with the BP and the existentially quantified sentence (6c), as in (7a)

3 The proposal put forward in Section 3, that BPs have a weak existential plain meaning and that their generic meaning arises through double strengthening, might open the way to new approaches to the sticky issues of generic BPs' actual quantificational force and tolerance to exceptions, at least for those cases where generic BPs behave differently than BPs bound by overt generic adverbs. 
No need for a dedicated theory of English bare plurals

and (7c). The triplet in (8) makes the same point for the DE environment provided by the nuclear scope of $f e w{ }^{4}$

(7) a. \#It's false that dogs are intelligent, but many of them are.

b. It's false that all dogs are intelligent, but many of them are.

c. \#It's false that some dogs are intelligent, but many of them are.

(8) a. Pretty much in every country, at least half of the men are brave. \#But only in a few countries, men are brave.

b. Pretty much in every country, at least half of the men are brave. But only in a few countries, all men are brave.

c. Pretty much in every country, at least half of the men are brave. \#But only in a few countries, some men are brave.

In conclusion, the G-inference disappears in DE environments, raising a compositionality puzzle: sentence (6a) does not mean the negation of sentence (5a), although syntactically the former is indeed just the negation of the latter.

Finally, let's consider the case of embedding in non-monotonic environments, illustrated in (9). Sentence (9a) with the BP differs from the existentially quantified sentence $(9 c)$, since only the latter sentence would feel true in a scenario where only half of the dogs turned out to be intelligent in the unique country mentioned here. In other words, the G-inference is visible in the UE component of the non-monotonic meaning. Furthermore, sentence (9a) with the BP differs from the universally quantified sentence $(9 \mathrm{~b})$, since only the latter sentence would feel true in a scenario where there are a few more countries where half of the dogs are intelligent. In other words, the G-inference is not visible in the DE component of the non-monotonic meaning.

(9) a. In exactly one country, dogs are intelligent.

b. In exactly one country, all dogs are intelligent.

c. In exactly one country, some dogs are intelligent.

(10) a. In exactly one country, dogs are intelligent ... \#And in two other countries dogs are sometimes intelligent.

b. In exactly one country, dogs are generally intelligent ... And in two other countries dogs are sometimes intelligent.

c. In exactly one country, dogs are sometimes intelligent ... \#And in two other countries dogs are sometimes intelligent.

The latter intuition concerning the DE component of the non-monotonic meaning is brought out in (10) by completing sentences (9) with the proper continuation.

4 The behavior of the G-inference in the case of the DE environment provided by the restrictor of universal quantifiers is admittedly more tricky and I have to leave the issue open at the moment. 
Giorgio Magri

\subsection{Summary}

The behavior (5)-(9) of the G-inference under embedding in environments of different monotonicity is analogous to the behavior (1)-(4) of the P-inference: the inference is visible in UE environments and disappears in DE environments, with both behaviors simultaneously displayed in the case of $\mathrm{UE}+\mathrm{DE}=$ non-monotonic environments. This analogy calls for a unified account.

\section{A unified account: extending Spector (2007) from the P- to the G-inference}

The behavior of the P-inference reviewed in Subsection 2.1 and the behavior of the G-inference reviewed in Subsection 2.2 have been accounted for with different tools in the literature. The behavior of the P-inference has been accounted for in terms of a competition between singular and plural morphology, cast within the framework of the theory of scalar implicatures (Spector 2007) or the closely related theory of Maximize Presupposition (Sauerland 2003). On the contrary, the behavior of the G-inference has traditionally been accounted for through a dedicated presupposition, called the Homogeneity presupposition (Fodor 1970; von Fintel 1997; Gajewski 2005).

Yet, the analogy between the two behaviors calls for a unified account. Should the implicature-based account for the P-inference be extended to the G-inference? Or vice versa, should the presupposition-based account for the G-inference be extended to the P-inference? In principle, both options are viable. In this Section, I develop the former option: I review Spector's (2007) implicature-based account for the P-inference and straightforwardly extend it to the G-inference. In Section 4, I will then argue in favor of this choice, by exploring in detail a peculiar prediction.

\subsection{Preliminaries on scalar implicatures}

Textbook semantics counterintuitively assumes sentence (11a) to be true in a scenario where John solved all of the problems. Since seminal work by Grice (1975), this assumption has been reconciled with intuitions as follows. Indeed, the plain meaning of sentence (11a) is (11b), whereby it would be true in a scenario where John solved all of the problems. Yet, the choice of the item some triggers the scalar implicature that he did not solve all of them. And the sentence together with its implicature effectively ends up equivalent to (11c), as desired.

(11) a. John solved some of the problems.

b. Joh solved at least some of the problems. $\quad=\llbracket(11 \mathrm{a}) \rrbracket$

c. John solved only some of the problems. $=[[[(11 \mathrm{a})]]$ 
No need for a dedicated theory of English bare plurals

The conjunction of the plain meaning of a sentence with its scalar implicatures is called its strengthened meaning. I use the standard notation $\llbracket \cdot \rrbracket$ for the plain meaning ${ }^{5}$ and the notation $[[[\cdot]]]$ for the strengthened meaning (for a mnemonic, think about your mobile phone: the more bars you have, the stronger the signal). In this Subsection, I introduce background assumptions on the strengthened meaning.

As illustrated in (11), the strengthened meaning [[[ $\varphi]]$ of a sentence $\varphi$ can be described as the plain meaning of the corresponding sentence with an overt only associating with the scalar item. Fox (2007) interprets this observation algorithmically, as in (12): the strengthened meaning of a sentence is obtained by appending to its LF a covert variant of only, called the exhaustivity operator and notated EXH.

$$
[\llbracket \varphi]]=\llbracket \mathrm{EXH} \varphi \rrbracket
$$

By (12), the theory of the strengthened meaning is reduced to the semantics of the exhaustivity operator EXH or its overt counterpart only. Many approaches assume that the exhaustivity operator EXH takes a prejacent proposition $\varphi$ and does two things, as in (13). First, it asserts the prejacent $\varphi$. Second, it negates a bunch of alternatives $\psi$, namely all the alternatives $\psi$ in the set $\operatorname{Exc}(\varphi)$ of alternatives excludable with respect to $\varphi$. Each conjunct $\neg \psi$ in (13) is called a scalar implicature.

$$
\llbracket \operatorname{EXH}(\varphi) \rrbracket=\llbracket \varphi \rrbracket \wedge \bigwedge_{\psi \in \operatorname{Exc}(\varphi)} \neg \llbracket \psi \rrbracket
$$

The set $\operatorname{Exc}(\varphi)$ of excludable alternatives is usually defined in two steps. To start, the set $\operatorname{Alt}(\varphi)$ of scalar alternatives of the prejacent $\varphi$ is defined as the set of those LFs that can be obtained from the target LF $\varphi$ by replacing one or more scalar items in $\varphi$ with their Horn-mates. I will come back in Subsections 3.2 and 3.3 to the proper definition of Horn-mateness. The set of excludable alternatives $\operatorname{Exc}(\varphi)$ is then defined as the subset of those scalar alternatives $\psi$ that can be negated consistently with the prejacent $\varphi$ (for instance, the alternatives $\psi$ that asymmetrically entail $\varphi$ ).

Fox (2007) (building on Kratzer \& Shimoyama 2002) and Spector (2007) modify the framework just sketched, by replacing assumption (12) with the variant in (14), that allows for an iterated exhaustivity operator.

$$
[\llbracket[\varphi]]=\llbracket \operatorname{EXH}(\operatorname{EXH}(\varphi)) \rrbracket
$$

As will become clear later on, the intuition behind the switch from (12) to (14) is as follows: according to the classical assumption (12), it is only the plain meaning $\llbracket \psi \rrbracket$ of the alternatives that plays a role in the computation of the strengthened meaning of the prejacent; the revised assumption (14) allows instead the strengthened meaning $[[[\psi]]]$ of the alternatives to play a role as well.

5 As is common in the literature, I often drop $\llbracket \cdot \rrbracket$, sloppily using the same symbol $\varphi$ for both an LF and its plain meaning. 
As a matter of fact, the two definitions (12) and (14) turn out to be actually equivalent in most cases. To illustrate, consider again the prejacent (11a), abbreviated as SOME in (15a). Consider furthermore the two alternatives obtained by replacing some with many and all, abbreviated as MANY and ALL in (15b) and (15c).
a. SOME $=$ John solved some of the problems.
b. MANY = John solved many of the problems.
c. $\mathrm{ALL}=$ John solved all of the problems.

Obviously, ALL asymmetrically entails MANY that in turn asymmetrically entails SOME as in (16), where the arrows stand for asymmetric entailment. Furthermore, the three items some, many, and all are usually assumed to be Horn-mates of each other as depicted in (17), where the dotted lines stand for Horn-mateness.

(16)

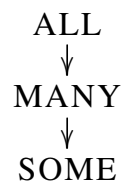

(17)

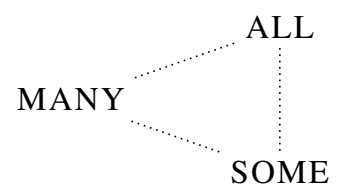

By the original assumption (12), the strengthened meaning of the prejacent SOME boils down to the conjunction of the prejacent SOME itself with the negation of its alternative MANY as in (18) (the alternative ALL can be ignored, because its negation is in turn entailed by the negation of the alternative MANY).

$$
[[[\operatorname{SOME}]]]=\operatorname{EXH}(\operatorname{SOME})=\operatorname{SOME} \wedge \neg \mathrm{MANY}
$$

By the revised assumption (14), we get the same result, but through the slightly more cumbersome computation (19). In step (a), I have unpacked through (13) the outer EXH: it asserts its prejacent $\mathrm{EXH}(\mathrm{SOME})$ and it negates the alternative EXH(MANY) obtained by replacing some with the Horn-mate many (again, the alternative $\operatorname{EXH}(\mathrm{ALL})$ can be ignored). In step (b), I have applied the definition (13) once more (again ignoring the alternative ALL in the computation of EXH(SOME) because it is irrelevant). Step (c) finally holds by logical equivalence.

$$
\begin{aligned}
{[[\operatorname{SOME}]] } & =\operatorname{EXH}(\operatorname{EXH}(\operatorname{SOME})) \stackrel{(a)}{=} \operatorname{EXH}(\operatorname{SOME}) \wedge \neg \operatorname{EXH}(\text { MANY }) \\
& \stackrel{(b)}{=}(\operatorname{SOME} \wedge \neg \mathrm{MANY}) \wedge \neg(\text { MANY } \wedge \neg \mathrm{ALL}) \stackrel{(c)}{=} \operatorname{SOME} \wedge \neg \text { MANY }
\end{aligned}
$$

This example shows that the iteration of EXH in (14) has no effects in standard cases. We need special patterns of entailment and Horn-mateness in order for iteration to have an effect. One such case is described by Spector (2007), as reviewed below.

\subsection{Spector's implicature-based account for the P-inference}

In Subsection 2.1, we have seen that the plural indefinite of sentence (20a) (abbreviated as PL) patterns as the singular indefinite of sentence (20c) (abbreviated 
No need for a dedicated theory of English bare plurals

as SING) in certain environments but as the quantified indefinite of sentence (20b) (abbreviated as TWO) in certain other environments.
a. PL $=$ John bought some books.
b. TWO = John bought (at least) two books.
c. SING $=$ John bought a book.

We have a clear understanding of the semantics and the pragmatics of the two sentences SING and TWO. With respect to their semantics, we know that TWO asymmetrically entails SING, as represented by the arrow in (21).

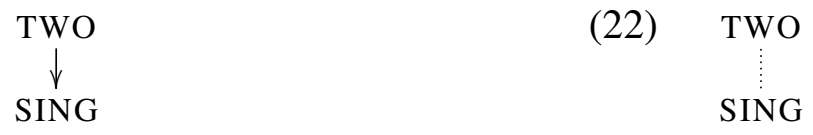

With respect to their pragmatics, we know that TWO is a scalar alternative of SING as represented by the dotted line in (22). The latter assumption is needed in order for SING to trigger the "only-one" implicature.

Now we want to fit sentence PL into the picture (21)-(22). Let's start with its semantics. The issue is tricky, as PL was found in Subsection 2.1 to behave as TWO in UE environments but as SING in DE environments. Spector (2007) assumes that plural morphology has a weak semantics equivalent to singular morphology, whereby PL and SING are logically equivalent, as stated in (23b). Both are thus asymmetrically entailed by TWO, as stated in (23a) and (23c).

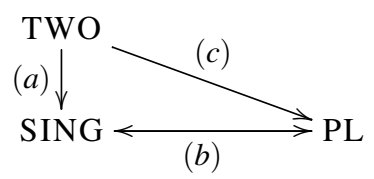

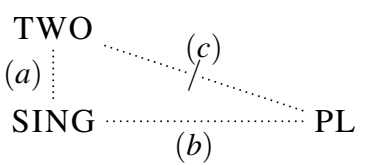

Furthermore, Spector assumes that PL and SING are scalar alternatives, as stated in (24b). Crucially, he assumes that, while SING is a scalar alternative of TWO as repeated in (24a), PL is not an alternative of TWO, as represented by the broken dotted line (24c). This is the only difference between SING and PL. Note that the diagram in (24) requires Horn-mateness not to be transitive.

Spector shows that the strengthened meaning computed through assumptions (13), (14), (23), and (24) accounts for the behavior of the P-inference under embedding documented in Subsection 2.1. To start with the case of UE environments, consider the unembedded sentence John bought some books, that was abbreviated as PL in (20a). We want its strengthened meaning to say that John bought at least two books. And that is indeed what we get, through the computation in (25). In step (25a), I have used the assumption (14) that the strengthened meaning is computed through an iterated exhaustivity operator. In step (25b), I have unpacked the outer EXH using (13) into the prejacent $\mathrm{EXH}(\mathrm{PL})$ and the negation of its unique alternative 
EXH(SING). In step (25c), I have unpacked the inner EXH. The alternative SING is not excludable relative to PL because it is equivalent to it, so that $\operatorname{EXH(PL)~boils~down~}$ to just PL. The alternative TWO is excludable relative to SING, so that EXH(SING) boils down to SING $\wedge \neg$ TWO. By the equivalence in (25d), the strengthened meaning says that John bought at least two books.

$$
\begin{aligned}
{[[\mathrm{PL}]]] } & \stackrel{(a)}{=} \operatorname{EXH}(\mathrm{EXH}(\mathrm{PL})) \stackrel{(b)}{=} \operatorname{EXH}(\mathrm{PL}) \wedge \neg \operatorname{EXH}(\mathrm{SING}) \\
& \stackrel{(c)}{=} \mathrm{PL} \wedge \neg(\mathrm{SING} \wedge \neg \mathrm{TWO}) \stackrel{(d)}{=} \mathrm{TWO}
\end{aligned}
$$

The intuition behind computation (25) can be made explicit as follows. The two alternatives SING and PL have the same plain meaning, by (23). They only differ because of their alternatives, by (24). In particular, SING is Horn-mate of TWO and therefore triggers the uniqueness implicature that $\neg$ TWO, contrary to PL. As this is the only difference between SING and PL, the only reason why the speaker has chosen PL over SING is that this uniqueness implicature that would have been triggered by SING is false. And the plurality inference triggered by PL is due to the negation of the uniqueness implicature that would have been triggered by SING.

So far, I have looked at UE environments. Nothing interesting happens in DE environments such as negation. Neither [not SING] nor [not PL] get strengthened. Thus, the intuitive equivalence between these two sentences follows straightforwardly from the equivalence of their plain meanings, postulated in (23b). In other words, pragmatic effects are wiped out in DE environments, where we can therefore probe into the actual plain semantics of singular and plural morphology. A computation analogous to (25) derives the desired meaning for non-monotonic environments.

\subsection{A straightforward extension of Spector's account to the G-inference}

In Subsection 2.2, we have seen that sentence BP in (26a) with a bare plural patterns as the existentially quantified sentence SOME in (26b) in certain environments but as the universally quantified sentence ALL in (26c) in certain other environments.
a. $\mathrm{BP}=$ Dogs are intelligent.
b. SOME $=$ Some dogs are intelligent.
c. $\mathrm{ALL}=\mathrm{All}$ dogs are intelligent.

More precisely, we have seen that the behavior relative to embedding displayed by the three sentences in (26) is formally analogous to the behavior displayed by the three sentences in (20), through the formal correspondences in (27).

$$
\mathrm{BP} \longleftrightarrow \mathrm{PL} \quad \mathrm{SOME} \longleftrightarrow \text { SING } \quad \text { ALL } \longleftrightarrow \text { TWO }
$$

In Subsection 3.2, we have seen that the behavior under embedding of the three sentences (20) follows from the pragma-semantic assumptions (23)-(24). Hence, the 
No need for a dedicated theory of English bare plurals

analogous behavior of the three sentences (26) follows from assumptions (28)-(29), formally analogous to (23)-(24) through the correspondences in (27).

(28)

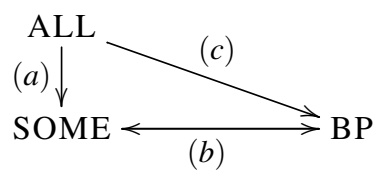

(29)

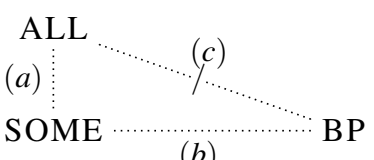

With respect to the semantics, sentence BP with the bare plural is logically equivalent to the existential sentence SOME, by (28b). In other words, bare plurals always contribute an existential plain meaning, no matter their readings. And both sentences BP and SOME are asymmetrically entailed by the universally quantified sentence ALL, as stated in (28a) and (28c). With respect to the pragmatics, SOME and ALL are of course alternatives, as stated in (29a). Furthermore, BP and SOME are alternatives of each other, as stated in (29b). The crucial difference between these two alternatives is that BP, contrary to SOME, is not an alternative to ALL, as stated in (29c).

The facts observed in Subsection 2.2 now follow straightforwardly. The existentially quantified sentence SOME triggers the implicature that the corresponding universally quantified sentence ALL is false, whereby the "only-some" inference. Again through double strengthening, sentence BP triggers the "not-only-some" implicature that the latter "only-some" implicature is false, whereby the generic/universal reading. The details of the reasoning are spelled out in the computation in (30), which is completely analogous to the one in (25).

$$
\begin{aligned}
{[[\mathrm{BP}]] } & =\operatorname{EXH}(\operatorname{EXH}(\mathrm{BP}))=\operatorname{EXH}(\mathrm{BP}) \wedge \neg \operatorname{EXH}(\mathrm{SOME}) \\
& =\mathrm{BP} \wedge \neg(\operatorname{SOME} \wedge \neg \mathrm{ALL})=\mathrm{ALL}
\end{aligned}
$$

In DE environments, there are no implicatures, so that bare plurals reveal their plain existential semantics (28b). Finally, a computation analogous to (30) derives the desired meaning also for the case of embedding in non-monotonic environments.

\section{Deriving a strengthened version of Diesing's correlation}

Assume that all arguments of a verb V are generated within VP, whereby they can or must move out. Diesing (1992: Ch. 2 and 3) then correlates the readings of BPs (generic or existential) and indefinites (presuppositional or non-presuppositional) with their syntactic position (outside of or within VP) as in (31) and (32).

A BP has a generic (existential) reading iff it is QRed outside of (left in situ within) VP at LF.

(32) An indefinite has a presuppositional (non-presuppositional) reading iff it is QRed outside of (left in situ within) VP at LF.

Since both (31) and (32) are double implications with the same right hand side, they can be collapsed into the corollary (33). 
(33) A BP has a generic (existential) reading in a certain LF iff an indefinite in the same position has a presuppositional (non-presuppositional) reading.

The distinction between presuppositional and non-presuppositional indefinites invoked in (32) was first made by Milsark (1977), developed by Diesing (1992), questioned by Reinhart (1995, 2006) and finally defended by von Fintel (1998). Based on the latter reference, I take the distinction between the two types of indefinites to be warranted. Yet, what is the presupposition triggered by presuppositional indefinites? Diesing addresses this question in the following passage (pp. 59-60):

"In [the sentence SOME ghosts are in the pantry; the others are in the attic], the determiner is stressed, and the presuppositional reading is most salient. This sentence presupposes the existence of ghosts. If no ghosts exist, [...] its truth-value will be undefined. The presuppositional reading, unlike the cardinal reading, can be paraphrased as a partitive: three of the ghosts."

At the beginning of the passage, Diesing suggests that what makes some ghosts presuppositional is the fact that it triggers the existential presupposition that "there exist ghosts". At the the end of the passage, she further clarifies her intuition, suggesting that what makes the indefinite some ghosts presuppositional is the fact that it is equivalent to the partitive some of the ghosts. But the presupposition carried by the partitive is stronger than plain existential: it presupposes that the relevant totality of ghosts denoted by the embedded definite is "given" or "familiar". Based on Diesing's discussion in Chapter 3, I submit that this tension between these two different construals of the presupposition triggered by presuppositional indefinites should be resolved in favor of the latter: the indefinite some ghosts is presuppositional because it is equivalent to the partitive some of the ghosts, whereby the totality of ghosts is given or familiar. ${ }^{6}$

Plausibly, it is precisely the indefinites in partitive constructions such as some of the ghosts that trigger the "only-some" scalar implicature that the corresponding alternative all of the ghosts is false. Indeed, this "only-some" implicature concerns the totality of the ghosts, and thus it makes no sense to derive this implicature if the totality of ghosts is not a given discourse entity. Based on these considerations, I thus restate Diesing's generalization (33) somewhat more explicitly as in (34).

6 von Fintel (1998) instead resolves the tension between the two options (just an existential presupposition versus a partitive presupposition) in favor of the former: "Note another issue: the presupposition that Diesing detects is not simply one of existence of entities that satisfy the common noun predicate of the indefinite. Both of her examples that we have seen so far [...] were described as involving presupposed existence of a particular set of ghosts or cellists. I will assume that we have here an interaction between a simple existence presupposition and the possibility of covert domain restriction". 
No need for a dedicated theory of English bare plurals

(34) A BP has a generic (existential) reading in a certain LF iff an indefinite in the same position triggers (does not trigger) the "only-some" implicature.

The extension (28)-(29) of Spector's pragma-semantics to BPs straightforwardly predicts Diesing's correlation (34). Let me make this point explicit. According to the semantics (28), a BP has a plain existential meaning. That will be its final meaning, unless that plain meaning gets strengthened into a generic/universal meaning. According to the pragmatics (29), this strengthening comes about through the negation of the "only-some" implicature of the corresponding indefinite. Of course, if the corresponding indefinite triggers no "only-some" implicature, then there is nothing to negate and thus no strengthening of the existential meaning of BPs into a generic/universal meaning. In other words, the proposed pragma-semantics (28)-(29) straightforwardly predicts the generic reading of a BP to be parasitic on the "only-some" implicature of the corresponding indefinite. And this prediction is borne out, as stated in Diesing's corollary (34). To substantiate this point, in Subsection 4.1 I go through various well known cases that illustrate this correlation.

Actually, the extension (28)-(29) of Spector's pragma-semantics to BPs derives a stronger version of Diesing's correlation (34) between BPs and overt indefinites. For Diesing, that correlation is an epiphenomenon of the two generalizations (31) and (32). And the latter two generalizations are independent of each other: the interpretation mechanism for generic BPs is independent of the interpretation mechanism for presuppositional indefinites, although both just happen to be sensitive to the same LF landmarks (the left edge of VP). According to the proposal developed here instead, the interpretation mechanism for generic BPs is completely parasitic on the interpretation mechanism for overt indefinites, more precisely on their implicatures. Thus, I predict not only that the availability of BPs' generic/universal reading should depend on the availability of the "only-some" implicature of indefinites, as stated in (34), but also that the quality of that generic/universal reading should depend on the quality of the "only-some" implicature triggered by the corresponding indefinite. In Subsection 4.2, I provide some initial evidence that this stronger correlation holds, by looking at Condoravdi's (1997) third reading and its distribution.

\subsection{Deriving Diesing's correlation}

The readings of BP subjects are well known to correlate with predicate type along the individual-level versus stage-level predicate (ILP/SLP) distinction (Carlson 1977). For instance, the BP subject dogs has a generic reading with the ILP carnivorous in (35a) which is unavailable in the case of the SLP play in (35b).

a. Dogs are carnivorous.

GENERIC

b. Dogs were playing in the backyard.

GENERIE 
Now consider the corresponding sentences with the BP dogs replaced with the overt indefinite some $\operatorname{dogs}$, as in (36). Out of the blue, (36a) triggers the "only-some" implicature, while (36b) does not.
a. Some dogs are carnivorous.
ONLY-SOME
b. Some dogs were playing in the backyard.
ONLY-SOME

The availability of the generic reading of the BP in (35) thus correlates with the availability of the "only-some" implicature of the indefinite in (36), as stated in the generalization (34) and predicted by the pragma-semantics in Subsection 3.3.

BP subjects of SLPs can be construed both existentially and generically. And the available reading often depends on whether the BP is parsed into the focus or the topic of the sentence (Wilkinson 1991). For instance, the BP subject firemen of the SLP available in (37) can be construed both generically and existentially, depending on whether the sentence is construed as being about firemen (37a) or about people available at the information desk over there (37b). Analogously, the BP subject typhoons of the habitual SLP arise in (38) can be construed both generically and existentially, depending on whether the sentence is construed as being about typhoons (40a) or about this part of the Pacific (40b).

(37) Firemen are usually available.

a. Talking about firemen...

GENERIC

b. Talking about who is available at the information desk...

GENERIC

(38) Typhoons arise in this part of the Pacific.

a. Talking about typhoons...

GENERIC

b. Talking about things that happen in this part of the Pacific... GENERIC

(39) Some firemen are usually available.

a. Talking about firemen...

ONLY-SOME

b. Talking about who is available at the information desk...

ONLY SOME

(40) Some typhoons arise in this part of the Pacific.

a. Talking about typhoons...

ONLY-SOME

b. Talking about things that happen in this part of the Pacific... ONLY-SOME

In conformity with the generalization (34), the corresponding sentences (39)-(40) with the BPs replaced by overt indefinites do or do not trigger the "only-some" implicature, depending on how the topic of these sentences is construed.

The case of BP objects is well known to be different from that of BP subjects. Both BP objects in (41a) and (41b) are interpreted existentially, although they occur with a SLP and an ILP respectively. Yet, some objects of ILPs do require a generic interpretation, as illustrated in (41c). 
No need for a dedicated theory of English bare plurals

a. John bought books.

GENERIC READING

b. John knows good lawyers.

GENERIC READING

c. John hates/loves lawyers.

GENERIC READING

Now consider the corresponding sentences with the BPs replaced with overt indefinites, as in (42). Out of the blue, (42c) triggers the "only-some" implicature that John hates/loves only some lawyers, not all of them. The corresponding implicature seems missing in the case of sentences (42a) and (42b).
a. John bought some books.
ONLY-SOME IMPLIC
b. John knows some good lawyers.
ONLY-SOME IMPLIC
c. John hates/loves some lawyers.
ONLY-SOME IMPLIC

Although the distribution of readings of BP objects is more intricate than that of BP subjects, the pattern of readings again conforms to the generalization (34) and thus follows from the pragma-semantics proposed in Subsection 3.3.

Existential BPs have been noticed to only take narrow scope relative to other scope bearing elements (Carlson 1977). ${ }^{7}$ A classical illustration of this generalization is provided by sentence (43). The BP firemen can have both generic and existential reading, as already recalled above for (37). Yet, the existential reading is crucially condemned to have narrow scope relative to the universal adverb. Namely, the sentence cannot mean that there are certain firemen that are usually available. Usually, this generalization is captured by constraining the scope of whatever interpretation mechanism is responsible for existential BPs. For instance, Diesing (1992) derives existential BPs through an operation of Default Existential Closure (DEC), and she therefore assumes it to have narrowest (i.e., VP) scope. Chierchia (1995) derives existential BPs through Derived Kind Predication (DKP), which he assumes is a "last-resort" type shifter, and is therefore only triggered in the most embedded position. No such approach is available to me, as I have assumed in Subsection 3.3 that BPs have existential force on their own.

\section{(43) Firemen are usually available.}

Yet, consider the corresponding sentence (44) with the BP firemen replaced with an overt indefinite some firemen. The indefinite can take both wide and narrow scope

7 Existential BPs have been documented to be able to take a scope narrower than what is possible for plain indefinites (Carlson 1977). This finding is perfectly compatible with the pragma-semantics proposed in Subsection 3.3. Furthermore, that proposal predicts the extreme narrow scope to be possible for existential BPs but not for generic ones: if the BP can sit in a very embedded position where an indefinite cannot sit, then it must receive its plain existential meaning, as there is no alternative that could trigger a "only-some" implicature and thus strengthen the BP into a generic/universal meaning. 
relative to the universal verb usually, as indicated by the paraphrases in (44a) and (44b). The two paraphrases behave quite differently relative to the availability of the "only-some" implicature, which is present for the wide-scope paraphrase (44a), not for the narrow-scope paraphrase (44b).

Some firemen are usually available.

a. Some firemen are such that they are usually available.

ONLY-SOME

b. Usually, some firemen are available (at the information desk). ONLY-SOME

The restriction of existential BPs to the narrowest scope can thus be accounted as follows within the pragma-semantics proposed in Subsection 3.3. The BP always has an existential plain meaning, with no need for additional mechanisms such as DEC or DKP. And BPs are allowed to take either wide or narrow scope, just as any other indefinites. Crucially, it looks like existential BPs cannot take wide scope because when they do, their plain existential meaning gets enriched into a generic/universal one, that masks the plain existential reading. This is due to the fact that the corresponding indefinite in a wide scope configuration like (44a) triggers an "only-some" implicature that the BP negates, yielding a "not-only-some" implicature. It is only in the narrow scope configuration that BPs are allowed to display their plain existential meaning, due to the fact that the corresponding indefinite does not trigger the "only-some" implicature from that position (44b), so that no pragmatic strengthening happens.

As illustrated in (35), BP subjects of ILPs only get the generic reading. Yet, the existential reading becomes surprisingly available when the BP is embedded under another universal operator (Fox 1995). For instance, sentence (45) can be construed as saying that for every man there are women related to him, so that the BP subject women is interpreted existentially in the scope of the universal quantifier every man.

$$
\text { Jewish women are related to every Jewish man. }
$$

GENERIC

$$
\text { Some jewish women are related to every Jewish man. }
$$

ONLY-SOME

In conformity with generalization (34), the corresponding sentence (46) with the BP Jewish women replaced by the indefinite some Jewish women triggers no implicature when scoped underneath the universal quantifier every Jewish man.

Finally, there are some ILPs whose BP subjects manage to surprisingly carry the existential reading (Fernald 2000). A couple of examples are provided in (47).

a. Italians own this restaurant.

GENERIC

b. Cities lie at the bottom of the mountains.

GENERIC

Once more, consider the corresponding sentences with the BPs replaced by overt indefinites, as in (48). 
No need for a dedicated theory of English bare plurals

(48) a. Some Italians own this restaurant.

ONLY-SOME

b. Some cities lie at the bottom of the mountain.

ONLY-SOME

There is clearly a way to construe these sentences (48) so that they trigger no "onlysome" implicature, so that the pattern (47)-(48) conforms to generalization (34) and thus follows from the pragma-semantics proposed in Subsection 3.3.

\subsection{Strengthening Diesing's correlation}

The BP subject students in (49a) is neither generic nor existential. Rather, it is equivalent to the definite the students (Condoravdi 1997).

(49) In 1985 there was a ghost haunting the campus...

a.... Students were aware of this fact.

b. ... Some students were aware of this fact.

Again, this interpretation is predicted by the pragma-semantics developed in Subsection 3.3. In fact, the reading predicted for this BP is existential (plain meaning) plus the negation of the implicature (if any) triggered by some students in the corresponding sentence (49b) (strengthening). Indeed, the indefinite in the latter sentence (49b) does trigger an implicature, so that the BP of the target sentence (49a) is correctly predicted not to have just a plain existential meaning. Crucially though, the implicature triggered by the indefinite in (49b) is not that it is false that students in general were aware of the ghost, so that the target sentence (49a) is not incorrectly predicted to be strengthened into a generic meaning ("It is a general property of students that they are/were aware of the ghost"). On the contrary, the implicature triggered by the indefinite in (49b) says that it is false that all of the students on campus were aware of the ghosts, so that the target sentence (49a) is predicted to have the intended meaning, through the negation of the latter implicature.

This third reading of BPs detected by Condoravdi seems to be constrained in subtle ways (Krifka, Pelletier, Carlson, ter Meulen, Link \& Chierchia 1995). Indeed, compare the BP students in (49a) with the BP lions in (50a). Despite the fact that the two cases look analogous, the former can be interpreted as a definite (the students on campus) while the latter cannot: sentence (50a) necessarily says something about lions in general, not about the lions in the cage.

(50) In this cage there are lions and tigers.

a. Lions are sick.

b. \#Some lions are sick.

This contrast follows from the pragma-semantics developed in Subsection 3.3. In fact, the corresponding sentence $(50 \mathrm{~b})$ with the $\mathrm{BP}$ lions replaced by the indefinite 
some lions feels degraded relative to the corresponding partitive Some of the lions are sick. This case is thus different from (49b), which is just as fine as the corresponding partitive Some of the students were aware.... I interpret the awkwardness of sentence (50b) in the context considered as due to the fact that it triggers the scalar implicature that not all lions (in the world) are sick, which does not fit with the topic of the discourse, plausibly restricted to the lions in the cage. The corresponding BP lions in (50a) is thus correctly predicted to be strengthened to a generic reading.

In Subsection 4.1, I have reviewed correlations between the distribution of BP's generic reading and the distribution of the "only-some" implicature triggered by presuppositional indefinites. This distributional correlation can be captured in a syntactic framework such as the one developed by Diesing, whereby the two distributions are derived though independent mechanisms that happen to be sensitive to the same LF landmarks. But this Subsection has argued that the correlation between BPs and the "only-some" implicature is stronger: it concerns not only the distribution of these readings but also their quality, namely whether they have the quality of generics or of Condoravdi's definites. The latter correlation does not follow from Diesing's system, while it is straightforwardly predicted by the pragma-semantics of Subsection 3.3.

\section{Conclusions}

In this paper, I have looked at two classes of facts. One class of facts concerns the distribution of the readings of English BPs, which is sensitive to the monotonicity of the environment (as reviewed in Subsection 2.2) as well as to a variety of other structural and lexical properties (as reviewed in Section 4). The other class of facts concerns the distribution of the "only-some" implicature triggered by indefinites, which again is sensitive to the monotonicity of the environment (e.g., implicatures disappear in DE environments) as well as to a variety of other structural and lexical properties (as reviewed in Section 4). The two distributions seem to be highly correlated - as already recognized by Diesing (1992), if we interpret her presuppositional indefinites as those that have a partitive meaning and therefore trigger the "only-some" implicature. In this paper, I have offered no account for the distribution of the "only-some" implicature triggered by indefinites. Yet, I have suggested that the correlation between the two distributions should be taken at face value. And I have thus derived the distribution of readings of BPs from the distribution of partitive/presuppositional indefinites and their "only-some" implicature, through a straightforward application of Spector's (2007) double strengthening mechanism. From this perspective, the crucial research question is how to explain the distribution of the "only-some" implicature triggered by indefinites, while there is no need for a dedicated theory of the distribution of readings of English BPs. 
No need for a dedicated theory of English bare plurals

\section{References}

Carlson, Gregory N. 1977. Reference to kinds in English: University of Massachusetts at Amherst dissertation. Published in 1980 by Garland Press, New York.

Chierchia, Gennaro. 1995. Individual-level predicates as inherent generics. In Gregory N. Carlson \& Francis Jeffry Pelletier (eds.), The Generic Book, 125175. The Univ. of Chicago Press.

Condoravdi, Cleo. 1997. Descriptions in contexts: Yale University dissertation. Published 1997 by Garland Press, New York.

Diesing, Molly. 1992. Indefinites. Cambridge, Mass.: MIT Press.

Fernald, Theodore B. 2000. Predicates and temporal arguments. New York, Oxford: Oxford University Press.

von Fintel, Kai. 1997. Bare plurals, bare conditionals, and only. Journal of Semantics 14. 1-56.

von Fintel, Kai. 1998. Evidence for presuppositional indefinites. MIT manuscript.

Fodor, Janet Dean. 1970. The linguistic description of opaque contexts: MIT dissertation.

Fox, Danny. 1995. Economy and scope. Natural Language Semantics 3. 283-341.

Fox, Danny. 2007. Free choice and the theory of scalar implicatures. In Uli Sauerland \& Penka Stateva (eds.), Presupposition and implicature in compositional semantics, 71-120. New York: Palgrave Macmillan.

Gajewski, Jon. 2005. Neg-raising: Polarity and presupposition: MIT dissertation.

Grice, Paul. 1975. Logic and conversation. In Peter Cole \& Jerry Morgan (eds.), Syntax and Semantics 3: Speech acts, 41-58. New York: Academic Press.

Heim, Irene. 1988. The semantics of definite and indefinite noun phrases: University of Massachusetts at Amherst dissertation.

Kratzer, Angelika \& Junko Shimoyama. 2002. Indeterminate pronouns: The view from Japanese. In Y. Otsu (ed.), Proceedings of the 3rd Tokyo Conference on Psycholinguistics, Hituzi Syobo, Tokyo.

Krifka, Manfred, Francis Jeffry Pelletier, Gregory N. Carlson, Alice ter Meulen, Godehard Link \& Gennaro Chierchia. 1995. Genericity: An introduction. In Gregory N. Carlson \& Francis Jeffry Pelletier (eds.), The Generic Book, 1-124. Chicago and London: University of Chicago Press.

Milsark, Gary L. 1977. Toward an explanation of certain peculiarities of the existential. Linguistic Analysis 3(1). 1-29.

Reinhart, Tanya. 1995. Interface strategies. OTS, Utrecht University.

Reinhart, Tanya. 2006. Interface strategies. Optimal and costly computations. Cambridge, MA: MIT Press.

Sauerland, Uli. 2003. A new semantics for number. In Robert B. Young \& Yuping 
Zhou (eds.), Proceedings of Semantics and Linguistic Theory (SALT) 13, 258275.

Spector, Benjamin. 2007. Aspects of the pragmatics of plural morphology: On higher-order implicatures. In Uli Sauerland \& Penka Stateva (eds.), Presuppositions and implicatures in compositional semantics, 243-281. PalgraveMacmillan.

Wilkinson, Karina. 1991. Studies in the semantics of generic noun phrases: University of Massachusetts, Amherst dissertation.

Giorgio Magri

LLF UMR 7110

CNRS and University Paris-Diderot

5 , rue Thomas Mann

F-75205 Paris Cedex 13

magrigrg@gmail.com 